PROCEEDINGS OF THE

AMERICAN MATHEMATICAL SOCIETY

Volume 125, Number 10, October 1997, Pages 2875-2882

S 0002-9939(97)03968-3

\title{
ON THE RANKS OF SINGLE ELEMENTS OF REFLEXIVE OPERATOR ALGEBRAS
}

\author{
W. E. LONGSTAFF AND ORESTE PANAIA
}

(Communicated by Palle E. T. Jorgensen)

\begin{abstract}
For any completely distributive subspace lattice $\mathfrak{L}$ on a real or complex reflexive Banach space and a positive integer $n$, necessary and sufficient (lattice-theoretic) conditions are given for the existence of a single element of $A l g \mathfrak{L}$ of rank $n$. Similar conditions are given for the existence of single elements of infinite rank. From this follows a relatively simple lattice-theoretic condition which characterises when every non-zero single element has rank one. Slightly stronger results are obtained for the case where $\mathfrak{L}$ is finite, including the fact that every single element must then be of finite rank.
\end{abstract}

\section{INTRODUCTION}

An element $s$ of an (abstract) algebra $\mathcal{A}$ is called single if, whenever $a s b=0$ with $a, b \in \mathcal{A}$, then $a s=0$ or $s b=0$. It is easy to show that an operator of rank one is a single element of any operator algebra containing it. It is primarily for this reason that the notion of 'single element' plays a role in the representation theory of $C^{*}$-algebras, or more generally, of semisimple Banach algebras, and also in the study of algebraic homomorphisms between reflexive operator algebras (see [3, 4, 5] for some results and further references).

If $\mathfrak{L}$ is a completely distributive subspace lattice (see definition below) on a real or complex normed space, then Alg $\mathfrak{L}$ contains a plethora of rank one operators (so many in fact, that their set of invariant subspaces is $\mathfrak{L}[2,6]$ ). Earlier results of Ringrose [8] and Lambrou [3] show that if $\mathfrak{L}$ is a nest, respectively, an atomic Boolean algebra, then every non-zero single element of Alg $\mathfrak{L}$ has rank one. As mentioned in [4], Moore showed that this need not always be the case and asked if there was a simple lattice-theoretic condition on $\mathfrak{L}$ both necessary and sufficient for every non-zero single element of $A l g \mathfrak{L}$ to have rank one. An affirmative answer to Moore's question is presented below. A systematic study of single elements of reflexive algebras of the type $A l g \mathfrak{L}$, where $\mathfrak{L}$ is completely distributive, was initiated by Lambrou in [4]. Amongst many other interesting results and examples, he shows that single elements of infinite rank are possible. The present note owes much to the ideas contained in [4] and is a contribution to the study initiated by it. For the sake of simplicity the underlying space is taken to be a reflexive Banach space.

Given a positive integer $n$ we show (Theorem 1 ) that the existence of a single element of $A l g \mathfrak{L}$ of rank $n$ depends on a lattice-theoretic condition on $\mathfrak{L}$ which is

Received by the editors April 1, 1996.

1991 Mathematics Subject Classification. Primary 47C05.

(C)1997 American Mathematical Society 
relatively simple to state. Similar lattice-theoretic conditions are obtained (Theorem 2) for the existence of a single element of infinite rank. It immediately follows that if $A l g \mathfrak{L}$ contains a single element of rank $m$ (possibly infinite), then it contains a single element of rank $n$, for every $1 \leq n<m$. Finally (Theorem 3 ) a slightly stronger result is obtained for the case where $\mathfrak{L}$ is finite, including the fact that single elements must then be of finite rank.

\section{NotATION AND PRELIMINARIES}

Throughout what follows $X$ will denote a real or complex reflexive Banach space, with topological dual $X^{*}$. The terms operator on $X$ and subspace of $X$ shall mean, respectively, bounded linear mapping of $X$ into itself, and closed linear manifold of $X$. If $T$ is an operator on $X$ its range is denoted by $\mathcal{R}(T)$. For non-zero vectors $e^{*} \in X^{*}$ and $f \in X$, the rank one operator defined by $x \mapsto e^{*}(x) f$ is denoted by $e^{*} \otimes f$. Clearly $\left(e^{*} \otimes f\right)^{*}=\hat{f} \otimes e^{*}$, where $\hat{f}$ is the image of $f$ under the canonical map of $X$ onto $X^{* *}$. The linear span of a vector $g$ is denoted $\langle g\rangle$.

If $\mathcal{F}$ is a collection of subspaces of $X, A l g \mathcal{F}$ denotes the set of operators on $X$ leaving every member of $\mathcal{F}$ invariant. Obviously $A l g \mathcal{F}$ contains the identity operator and it is not difficult to show that it is closed in operator-norm. Thus $A l g \mathcal{F}$ is a unital Banach algebra. A collection $\mathfrak{L}$ of subspaces of $X$ is called a subspace lattice on $X$ if it contains (0) and $X$, and is complete in the sense that it is closed under the formation of arbitrary closed linear spans (denoted ' $V$ ') and intersections. Operator algebras of the type $A l g \mathfrak{L}$, where $\mathfrak{L}$ is a subspace lattice, are called reflexive operator algebras.

If $L$ is a subspace of $X$, its annihilator is denoted by $L^{\perp}$. Thus $L^{\perp}=\left\{e^{*} \in X^{*} \mid\right.$ $e^{*}(f)=0$, for every $\left.f \in L\right\}$. Dually, if $M$ is a subspace of $X^{*}$, its pre-annihilator is denoted by ${ }^{\perp} M$. Thus ${ }^{\perp} M=\left\{f \in X \mid e^{*}(f)=0\right.$, for every $\left.e^{*} \in M\right\}$. We have $X^{\perp}=(0),(0)^{\perp}=X^{*}$ and ${ }^{\perp}\left(L^{\perp}\right)=L$. Also, since $X$ is reflexive, we have $\left({ }^{\perp} M\right)^{\perp}=M$. For every family $\left\{L_{\gamma}\right\}_{\gamma \in \Gamma}$ of subspaces of $X$, we have $\left(\bigvee_{\gamma \in \Gamma} L_{\gamma}\right)^{\perp}=$ $\bigcap_{\gamma \in \Gamma} L_{\gamma}^{\perp}$ and, again by reflexivity, $\left(\bigcap_{\gamma \in \Gamma} L_{\gamma}\right)^{\perp}=\bigvee_{\gamma \in \Gamma} L_{\gamma}^{\perp}$. Consequently, if $\mathfrak{L}$ is a subspace lattice on $X$, then $\mathfrak{L}^{\perp}=\left\{L^{\perp} \mid L \in \mathfrak{L}\right\}$ is a subspace lattice on $X^{*}$. Clearly $\operatorname{Alg} \mathfrak{L}^{\perp}=\left\{A^{*} \mid A \in \operatorname{Alg} \mathfrak{L}\right\}$.

An abstract lattice $Y$ is called distributive if $a \wedge(b \vee c)=(a \wedge b) \vee(a \wedge c)$ and its dual hold identically in $Y$. If $Y$ is complete with least element 0 and greatest element 1 , it is said to be complemented if, for every $a \in Y$ there exists $a^{\prime} \in Y$ such that $a \vee a^{\prime}=1$ and $a \wedge a^{\prime}=0$. An element $b \in Y$ is called an atom if $0 \leq c \leq b$, $c \in Y$ implies that $c=0$ or $c=b$. If every non-zero element of $Y$ is the join of the atoms it contains, then $Y$ is called atomic. A complete complemented distributive lattice is called a complete Boolean algebra. A complete totally ordered lattice is called a nest. A complete lattice $Y$ is called completely distributive if for every family $\left\{a_{\alpha, \beta}\right\}_{\alpha \in I, \beta \in J}$ of elements of $Y$

$$
\bigwedge_{\alpha \in I} \bigvee_{\beta \in J} a_{\alpha, \beta}=\bigvee_{f \in J^{I}} \bigwedge_{\alpha \in I} a_{\alpha, f(\alpha)}
$$

and its dual hold, where $J^{I}$ denotes the set of all maps from $I$ into $J$. Every nest and every complete atomic Boolean algebra is completely distributive. The formal definition of complete distributivity just given is, in practice, difficult to use. Alternative characterisations of complete distributivity have proven to be more useful (see $[6,7]$ ). For a complete lattice $Y$ the following are equivalent: 
(i) $Y$ is completely distributive.

(ii) $a=\bigwedge\left\{b_{-} \mid b \in Y\right.$ and $\left.b \not \leq a\right\}$, for every $a \in Y$.

(iii) $a=\bigvee\left\{c_{+} \mid c \in Y\right.$ and $\left.a \not \leq c\right\}$, for every $a \in Y$.

(iv) $a=\bigvee\left\{b \mid b \in Y\right.$ and $\left.a \not \leq b_{-}\right\}$, for every $a \in Y$.

Here $b_{-}=\bigvee\{x \mid x \in Y$ and $b \not \leq x\}$ and $c_{+}=\bigwedge\{y \mid y \in Y$ and $y \not \leq c\}$, for every $b, c \in Y$ (in any complete lattice the conventions $\bigvee \emptyset=0$ and $\bigwedge \emptyset=1$ are adopted).

If $\mathfrak{L}$ is a completely distributive subspace lattice on $X$, it is not too difficult to show that $\mathfrak{L}^{\perp}$ is also completely distributive (on $X^{*}$ ). Indeed, for every $M \in \mathfrak{L}$, $\left(M_{+}\right)^{\perp}=\left(M^{\perp}\right)_{-}$(where the '-' is computed in $\left.\mathfrak{L}^{\perp}\right)$. So, for every $L \in \mathfrak{L}, L=$ $\bigvee\left\{M_{+} \mid M \in \mathfrak{L}\right.$ and $\left.L \nsubseteq M\right\}$ gives $L^{\perp}=\bigcap\left\{\left(M^{\perp}\right)_{-} \mid M^{\perp} \in \mathfrak{L}^{\perp}\right.$ and $\left.M^{\perp} \nsubseteq L^{\perp}\right\}$. For such a subspace lattice $\mathfrak{L}$ we denote by $\mathcal{J}$ the set of elements $\mathcal{J}=\{J \in \mathfrak{L} \mid J \neq(0)$ and $\left.J_{-} \neq X\right\}$. The relevance of $\mathcal{J}$ is due to the following lemma which will be frequently used.

Lemma 1 (Longstaff [6] (see also [2])). If $\mathfrak{L}$ is a subspace lattice on a real or complex normed space, the rank one operator $e^{*} \otimes f$ belongs to Alg $\mathfrak{L}$ if and only if there is an element $J \in \mathcal{J}$ such that $f \in J$ and $e^{*} \in\left(J_{-}\right)^{\perp}$.

\section{Completely Distributive lattices}

Throughout this section $\mathfrak{L}$ will denote a completely distributive subspace lattice on a (real or complex, reflexive) Banach space $X$. First, given a positive integer $n$, we show how a single element of $A l g \mathfrak{L}$ of rank $n$ can be constructed, given that $\mathfrak{L}$ satisfies a relatively simple lattice condition. We then show that this lattice condition is necessary for the existence of such a single element.

Proposition 1. If $n$ is a positive integer and there exist sets of $\mathcal{J}$-elements $\left\{M_{i} \mid\right.$ $1 \leq i \leq n\}$ and $\left\{K_{j} \mid 1 \leq j \leq n\right\}$ of $\mathfrak{L}$ satisfying

(1) $M_{i} \cap M_{j}=(0)$, if $i \neq j$,

(2) $K_{i-} \vee K_{j-}=X$, if $i \neq j$,

(3) $\bigvee_{i=1}^{n} M_{i} \subseteq \bigcap_{j=1}^{n} K_{j}$,

then, for any choice of non-zero vectors $f_{i} \in M_{i}$ and $e_{j}^{*} \in\left(K_{j-}\right)^{\perp}, 1 \leq i, j \leq n$, the operator $S=\sum_{j=1}^{n} e_{j}^{*} \otimes g_{j}$, where $g_{1}=\sum_{k=1}^{n} f_{k}$ and $g_{j}=\left(\sum_{k=1 ; k \neq j}^{n} f_{k}\right)-f_{j}$,

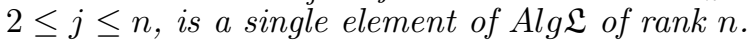

Proof. Note that $g_{j} \in K_{j}$, for every $1 \leq j \leq n$, so $e_{j}^{*} \otimes g_{j} \in A l g \mathfrak{L}$ by Lemma 1 . It follows that $S \in A l g \mathfrak{L}$. Since $M_{i} \cap\left(\bigvee_{j \neq i} M_{j}\right)=\bigvee_{j \neq i}\left(M_{i} \cap M_{j}\right)=(0)$, for every $1 \leq i \leq n$, the set of vectors $\left\{f_{i} \mid 1 \leq i \leq n\right\}$ is linearly independent. It readily follows that $\left\{g_{j} \mid 1 \leq j \leq n\right\}$ is linearly independent. Similarly $\left\{e_{j}^{*} \mid 1 \leq j \leq n\right\}$ is linearly independent since $\left(K_{j-}\right)^{\perp} \cap\left(\bigvee_{i \neq j}\left(K_{i-}\right)^{\perp}\right)=\bigvee_{i \neq j}\left(K_{j-}\right)^{\perp} \cap\left(K_{i-}\right)^{\perp}=(0)$, for every $1 \leq j \leq n$. Thus $S$ has rank $n$.

Let $A, B \in A \bar{l} g \mathfrak{L}$ satisfy $A S B=\sum_{k=1}^{n} B^{*} e_{k}^{*} \otimes A g_{k}=0$, and suppose that $A S=$ $\sum_{k=1}^{n} e_{k}^{*} \otimes A g_{k} \neq 0$. Then $A g_{j} \neq 0$, for some $1 \leq j \leq n$. In fact, $A g_{k} \neq 0$, for every $1 \leq k \leq n$. For, if $A g_{k}=0$, then, for every $1 \leq i \leq n, A f_{i} \in M_{i} \cap\left(\bigvee_{l \neq i} M_{l}\right)=(0)$, so $A f_{i}=0$. Now, for every $1 \leq j \leq n$, there exists $h_{j}^{*} \in X^{*}$ such that $h_{j}^{*}\left(A g_{j}\right) \neq 0$. So

$$
0=(A S B)^{*}\left(h_{j}^{*}\right)=\sum_{k=1}^{n} h_{j}^{*}\left(A g_{k}\right) B^{*} e_{k}^{*}
$$


gives

$$
B^{*} e_{j}^{*}=-\sum_{\substack{k=1 \\ k \neq j}}^{n} \frac{h_{j}^{*}\left(A g_{k}\right)}{h_{j}^{*}\left(A g_{j}\right)} B^{*} e_{k}^{*} .
$$

Thus $B^{*} e_{j}^{*} \in\left(K_{j-}\right)^{\perp} \cap\left(\bigvee_{k \neq j}\left(K_{k-}\right)^{\perp}\right)=(0)$, so $B^{*} e_{j}^{*}=0$, for every $1 \leq j \leq n$.

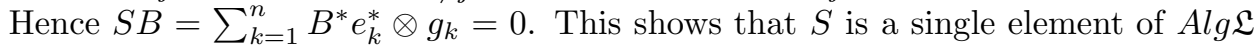
and the proof is complete.

Theorem 1. Let $\mathfrak{L}$ be a completely distributive subspace lattice on a reflexive Banach space $X$. If $n$ is a positive integer, then Alg $\mathfrak{L}$ contains a single element of rank $n$ if and only if there exist sets of $\mathcal{J}$-elements $\left\{M_{i} \mid 1 \leq i \leq n\right\}$ and $\left\{K_{j} \mid 1 \leq j \leq n\right\}$ of $\mathfrak{L}$ satisfying

(1) $M_{i} \cap M_{j}=(0)$, if $i \neq j$,

(2) $K_{i-} \vee K_{j-}=X$, if $i \neq j$,

(3) $\bigvee_{i=1}^{n} M_{i} \subseteq \bigcap_{j=1}^{n} K_{j}$.

Proof. The sufficiency of the condition follows from the preceding proposition.

Suppose that $A l g \mathfrak{L}$ contains a single element, $S$ say, of rank $n$. Since $\mathcal{J} \neq \emptyset$, we may suppose that $n>1$. By complete distributivity $X=\bigvee\{J \mid J \in \mathcal{J}\}(=$ $\bigvee\left\{L \mid L \in \mathfrak{L}\right.$ and $\left.\left.X \nsubseteq L_{-}\right\}\right)$. By [4, Lemma 3.2] $S(J)$ is at most one-dimensional, for every $J \in \mathcal{J}$. Thus $\mathcal{R}(S)=\bigvee\{S(J) \mid J \in \mathcal{J}\}$ and it follows that there is a basis $\left\{g_{j} \mid 1 \leq j \leq n\right\}$ of $\mathcal{R}(S)$ satisfying $S\left(K_{j}\right)=\left\langle g_{j}\right\rangle, 1 \leq j \leq n$, for some set of $\mathcal{J}$-elements $\left\{K_{j} \mid 1 \leq j \leq n\right\}$. Again by complete distributivity, $(0)=\bigcap\left\{J_{-} \mid\right.$ $J \in \mathcal{J}\}\left(=\bigcap\left\{L_{-} \mid L \in \mathfrak{L}\right.\right.$ and $\left.\left.L \nsubseteq(0)\right\}\right)$. Thus $X^{*}=\bigvee\left\{\left(J_{-}\right)^{\perp} \mid J \in \mathcal{J}\right\}$. By [4, Lemma 3.3] $S^{*}\left(J_{-}\right)^{\perp}$ is at most one-dimensional, for every $J \in \mathcal{J}$. Thus $\mathcal{R}\left(S^{*}\right)=\bigvee\left\{S^{*}\left(J_{-}\right)^{\perp} \mid J \in \mathcal{J}\right\}$ and it follows that there is a basis $\left\{e_{i}^{*} \mid 1 \leq i \leq n\right\}$ for $\mathcal{R}\left(S^{*}\right)$ satisfying $S^{*}\left(M_{i-}\right)^{\perp}=\left\langle e_{i}^{*}\right\rangle$, for $1 \leq i \leq n$, for some set of $\mathcal{J}$-elements $\left\{M_{i} \mid 1 \leq i \leq n\right\}$.

Let $1 \leq i \neq j \leq n$. Since $\bigcap\left\{J_{-} \mid J \in \mathcal{J}\right\}=(0)$, to show that $M_{i} \cap M_{j}=(0)$, it is enough to show that $M_{i} \cap M_{j} \subseteq J_{-}$, for every $J \in \mathcal{J}$. If this were not the case it would follow that for some $J \in \mathcal{J}, J \subseteq M_{i} \cap M_{j}$ so $J_{-} \subseteq M_{i-} \cap M_{j-}$. Then $\left(J_{-}\right)^{\perp}$ would contain both $\left(M_{i-}\right)^{\perp}$ and $\left(M_{j-}\right)^{\perp}$, and so $S^{*}\left(M_{i-}\right)^{\perp}=S^{*}\left(M_{j-}\right)^{\perp}=$ $S^{*}\left(J_{-}\right)^{\perp}$ (using [4, Lemma 3.3]). This would contradict the linear independence of $\left\{e_{i}^{*}, e_{j}^{*}\right\}$. Thus $M_{i} \cap M_{j}=(0)$. Continuing, since $\bigvee\{K \mid K \in \mathcal{J}\}=X$, to show that $K_{i-} \vee K_{j-}=X$, it is enough to show that $K \subseteq K_{i-} \vee K_{j-}$, for every $K \in \mathcal{J}$. Now $K$ cannot contain both $K_{i}$ and $K_{j}$ since otherwise $S\left(K_{i}\right)=S\left(K_{j}\right)=S(K)$ (using [4, Lemma 3.2]) and this would contradict the linear independence of $\left\{g_{i}, g_{j}\right\}$. Thus $K \subseteq K_{i-} \vee K_{j-}$ since either $K_{i} \nsubseteq K$ (so $K \subseteq K_{i-}$ ) or $K_{j} \nsubseteq K$ (so $K \subseteq K_{j-}$ ). Thus $K_{i-} \vee K_{j-}=X$.

Finally, we show that $\bigvee_{i=1}^{n} M_{i} \subseteq \bigcap_{j=1}^{n} K_{j}$. Let $1 \leq i, j \leq n$ and suppose that $M_{i} \nsubseteq K_{j}$. Choose $f \in K_{j}$ such that $S f \neq 0$ and choose $0 \neq e^{*} \in\left(K_{j-}\right)^{\perp}$. Further, choose $g^{*} \in\left(M_{i-}\right)^{\perp}$ such that $S^{*} g^{*} \neq 0$ and choose $0 \neq h \in M_{i}$. Then, by Lemma 1, each of $e^{*} \otimes f$ and $g^{*} \otimes h$ is a rank one operator of AlgL. Now $\left(g^{*} \otimes h\right) S=S^{*} g^{*} \otimes h \neq 0$ and $S\left(e^{*} \otimes f\right)=e^{*} \otimes S f \neq 0$. However, since $K_{j} \subseteq M_{i-}$, $f \in M_{i-}$ so $S f \in M_{i-}$. Hence

$$
\left(g^{*} \otimes h\right) S\left(e^{*} \otimes f\right)=g^{*}(S f)\left(e^{*} \otimes h\right)=0,
$$

and this contradicts the fact that $S$ is a single element. This completes the proof. 
Next we present the 'infinite' version of Theorem 1. Apart from modifications necessitated by the requirements of convergence, the proof is similar. For this reason only an abbreviated proof will be given.

Theorem 2. Let $\mathfrak{L}$ be a completely distributive subspace lattice on a reflexive $B a$ nach space $X$. The algebra Alg $\mathfrak{L}$ contains a single element of infinite rank if and only if there exist sets of $\mathcal{J}$-elements $\left\{M_{i} \mid i \geq 1\right\}$ and $\left\{K_{j} \mid j \geq 1\right\}$ of $\mathfrak{L}$ satisfying

(1) $M_{i} \cap M_{j}=(0)$, if $i \neq j$,

(2) $K_{i-} \vee K_{j-}=X$, if $i \neq j$,

(3) $\bigvee_{i=1}^{\infty} M_{i} \subseteq \bigcap_{j=1}^{\infty} K_{j}$.

Proof. First, suppose that the three conditions are satisfied by sets of $\mathcal{J}$-elements $\left\{M_{i} \mid i \geq 1\right\}$ and $\left\{K_{j} \mid j \geq 1\right\}$. For every $i \geq 1$ choose $f_{i} \in M_{i}$ such that $\left\|f_{i}\right\|=1 / 2^{i}$. Then $\left\{f_{i} \mid i \geq 1\right\}$ is linearly independent (by condition (1) and distributivity). For every $j \geq 1$ choose $e_{j}^{*} \in\left(K_{j-}\right)^{\perp}$ such that $\left\|e_{j}^{*}\right\|=1$. The series $\sum_{i=1}^{\infty} f_{i}$ is absolutely convergent so norm convergent. Put $\tilde{g}_{1}=\sum_{i=1}^{\infty} f_{i}$ and $\tilde{g}_{j}=\left(\sum_{k=1 ; k \neq j}^{\infty} f_{k}\right)-f_{j}$, for $j \geq 2$. Then, since $M_{i} \cap\left(\bigvee_{j \neq i} M_{j}\right)=(0)$, for every $i \geq 1, \tilde{g}_{j} \neq 0$, for every $j \geq 1$. Put $g_{j}=\tilde{g}_{j} /\left\|\tilde{g}_{j}\right\|$, for $j \geq 1$. The series $\sum_{j=1}^{\infty} \frac{e_{j}^{*} \otimes g_{j}}{2^{j}}$ is absolutely convergent so converges in operator norm. Denote its sum by $S$. Now $g_{j} \in K_{j}$, for every $j \geq 1$ (by condition (3)), so $e_{j}^{*} \otimes g_{j} \in A l g \mathfrak{L}$ by Lemma 1 . Hence $S \in A l g \mathfrak{L}$. Since $\mathfrak{L}^{\perp}$ is completely distributive, $\left(K_{i-}\right)^{\perp} \cap\left(\bigvee_{j \neq i}\left(K_{j-}\right)^{\perp}\right)=(0)$, for every $i \geq 1$, so $e_{i}^{*} \notin \bigvee_{j \neq i}\left(K_{j-}\right)^{\perp}$. By reflexivity and a corollary of the HahnBanach theorem, there exists a vector $x_{j} \in X$ such that $e_{i}^{*}\left(x_{j}\right)=\delta_{i j}$, for every $i, j \geq 1$. Then $S x_{j}=g_{j} / 2^{j}$ so $\tilde{g}_{j} \in \mathcal{R}(S)$, for every $j \geq 1$. Since $\tilde{g}_{j}=\tilde{g}_{1}-2 f_{j}$, for every $j \geq 2$, it follows that $f_{j} \in \mathcal{R}(S)$, for every $j \geq 2$. Hence $S$ has infinite rank.

The proof that $S$ is a single element of $A l g \mathfrak{L}$ follows in a similar manner, but with the obvious modifications, to that given in the proof of Proposition 1 (we still have: if $A \in A l g \mathfrak{L}$ and $A g_{k}=0$, for some $k \geq 1$, then $A g_{j}=0$, for every $j \geq 1$ ).

Conversely, suppose that $A l g \mathfrak{L}$ contains a single element, $S$ say, of infinite rank. Then, for every $J \in \mathcal{J}, S(J)=\left\langle g_{J}\right\rangle$, for some vector $g_{J}$ (possibly zero) and since $X=\bigvee\{J \mid J \in \mathcal{J}\}$ it follows that $\overline{\mathcal{R}(S)}=\bigvee\left\{\left\langle g_{J}\right\rangle \mid J \in \mathcal{J}\right\}$. By Zorn's Lemma $\left\{g_{J} \mid J \in \mathcal{J}\right\}$ has a maximal linearly independent subset which must be infinite since $S$ has infinite rank. Thus there exists a linearly independent set of vectors $\left\{g_{j} \mid j \geq 1\right\}$ of $X$ such that, for every $j \geq 1, S\left(K_{j}\right)=\left\langle g_{j}\right\rangle$, for some $K_{j} \in \mathcal{J}$. Similar considerations applied to $S^{*}$ give a linearly independent set of vectors $\left\{e_{i}^{*} \mid i \geq 1\right\}$ of $X^{*}$ such that, for every $i \geq 1, S^{*}\left(M_{i-}\right)^{\perp}=\left\langle e_{i}^{*}\right\rangle$, for some $M_{i} \in \mathcal{J}$. The proofs that conditions (1), (2) and (3) hold are almost identical to the corresponding arguments given in the proof of Theorem 1 . This completes the proof.

Remarks. 1. The operator $S$ constructed in the first part of the proof of the preceding theorem is, of course, compact. Thus, by the theorem, Alg $\mathfrak{L}$ contains a single element of infinite rank if and only if it contains a compact single element of infinite rank.

2. Combining Theorems 1 and 2 leads immediately to the following observations.

(i) If $m \in \mathbb{Z}^{+} \cup\{\infty\}$ and Alg $\mathfrak{L}$ contains a single element of rank $m$, then it contains a single element of rank $n$, for every $1 \leq n<m$.

(ii) In $A l g \mathfrak{L}$ every non-zero single element has rank one if and only if $A l g \mathfrak{L}$ does not contain a single element of rank 2 , if and only if the following is false: 
There exist $\mathcal{J}$-elements $M_{1}, M_{2}, K_{1}, K_{2}$ such that

(1) $M_{1} \cap M_{2}=(0)$,

(2) $K_{1-} \vee K_{2-}=X$,

(3) $M_{1} \vee M_{2} \subseteq K_{1} \cap K_{2}$.

This answers affirmatively the question, raised by Moore, mentioned in [4]. Note that, in particular, if $\mathfrak{L}$ is a nest or a complete atomic Boolean algebra (for which $\mathcal{J}$ is simply the set of atoms [6]), then it easily follows from the above that every non-zero single element of $A l g \mathfrak{L}$ has rank one. These facts were first observed, respectively, by Ringrose [8] and Lambrou [3]. Also, the above characterisation 'explains' Theorem 5.2 of [4] (by which non-zero single elements have rank one if $\mathfrak{L}$ is the ordinal sum of a nest and a completely distributive subspace lattice).

\section{Finite Distributive Lattices}

In the preceding section $\mathfrak{L}$ was a possibly infinite completely distributive subspace lattice (on a real or complex reflexive Banach space $X$ ). Throughout this section $\mathfrak{L}$ will be taken to be finite (and distributive). Note that, by Theorem 2, every single element of Alg $\mathfrak{L}$ has finite rank. A slight improvement to Theorem 1 is possible for this restricted class of subspace lattices as we will presently show. But first, some auxiliary results and definitions are required.

An element $a$ of an abstract lattice $Y$ is called join-irreducible if, whenever $a=b \vee c$ with $b, c \in Y$, then either $a=b$ or $a=c$. If $x \in Y$ has a representation $x=\bigvee_{j=1}^{n} x_{j}$ as a join of elements $x_{j} \in Y, 1 \leq j \leq n$, the element $x_{i}$ is called redundant (to this representation) if $x=\bigvee_{j=1 ; j \neq i}^{n} x_{j}$. If no element $x_{i}$ is redundant, this representation of $x$ is said to be irredundant. In any abstract finite distributive lattice every non-zero element has an irredundant representation as a join of nonzero join-irreducible elements. This representation is unique up to permutations of the non-zero join-irreducible elements. A proof of this result, due to Birkhoff, can be found in [1, Theorem 146, page 212].

Denote the set of non-zero join-irreducible elements of $\mathfrak{L}$ by $\mathcal{Z}$. Then $\mathcal{Z} \subseteq \mathcal{J}$. For if $L \in \mathcal{Z}$, then $L \nsubseteq L_{-}$since otherwise $L=L \cap L_{-}=\bigvee\{L \cap M \mid M \in \mathfrak{L}$ and $L \nsubseteq M$ \} would contradict join-irreducibility. Of course, the set of atoms of $\mathfrak{L}$ is non-empty and each atom belongs to $\mathcal{Z}$. Let $\left\{M_{i} \mid 1 \leq i \leq p\right\}$ be the set of atoms of $\mathfrak{L}$. Let $X=\bigvee_{j=1}^{q} K_{j}$ be the unique (up to permutations) irredundant representation of $X$ as a join of elements $\left\{K_{j} \mid 1 \leq j \leq q\right\}$ of $\mathcal{Z}$.

Proposition 2. The set of atoms of $\mathfrak{L}^{\perp}$ is $\left\{\left(K_{j-}\right)^{\perp} \mid 1 \leq j \leq q\right\}$ and $X^{*}=$ $\bigvee_{i=1}^{p}\left(M_{i-}\right)^{\perp}$ is the unique irredundant representation of $X^{*}$ as a join of non-zero join-irreducible elements.

Proof. Let $J^{\perp}$ be an atom of $\mathfrak{L}^{\perp}$. Then $J \neq X$ so $K_{j} \not \subseteq J$, for some $1 \leq j \leq q$. Thus $J \subseteq K_{j-}$ so $(0) \subset\left(K_{j-}\right)^{\perp} \subseteq J^{\perp}$ and so $J=K_{j-}$. On the other hand, each $\left(K_{k-}\right)^{\perp}$ is an atom of $\mathfrak{L}^{\perp}$. For, by irredundancy, $K_{k} \nsubseteq K_{i}$, for every $i \neq k$. Thus $\bigvee_{i \neq k} K_{i} \subseteq K_{k-}$. If $K \in \mathfrak{L}$ and $(0) \subset K^{\perp} \subseteq\left(K_{k-}\right)^{\perp}$, then since $K_{k-} \subseteq K$, $K_{i} \subseteq K$, for every $i \neq k$. Since $K \neq X$, it follows that $K_{k} \nsubseteq K$ so $K \subseteq K_{k-}$ and so $K^{\perp}=\left(K_{k-}\right)^{\perp}$.

Let $1 \leq i \leq p$. If $L \in \mathfrak{L}$ and $M_{i} \not \subseteq L$, then $(0) \subseteq M_{i} \cap L \subset M_{i}$ so $M_{i} \cap L=(0)$. It follows (using distributivity) that $M_{i} \cap M_{i-}=(0)$. Thus $\left(M_{i-}\right)^{\perp} \neq(0)$. Let $M, N \in \mathfrak{L}$ with $\left(M_{i-}\right)^{\perp}=M^{\perp} \vee N^{\perp}$ and suppose that $\left(M_{i-}\right)^{\perp} \neq M^{\perp}, N^{\perp}$. Then 
$M_{i-}=M \cap N$ and $M \nsubseteq M_{i-}, N \nsubseteq M_{i-}$. Thus $M_{i} \subseteq M \cap N$ so $M_{i} \subseteq M_{i-}$. This contradicts the fact that $M_{i} \cap M_{i-}=(0)$. Hence $\left(M_{i-}\right)^{\perp}$ is a non-zero joinirreducible element of $\mathfrak{L}^{\perp}$.

Now since $\bigcap_{i=1}^{p} M_{i-}$ contains no atom it is (0), so $X^{*}=\bigvee_{i=1}^{p}\left(M_{i-}\right)^{\perp}$. Finally we show that this join-representation of $X^{*}$ is irredundant. If it were not, then $\left(M_{i-}\right)^{\perp} \subseteq \bigvee_{j \neq i}\left(M_{j-}\right)^{\perp}$, for some $1 \leq i \leq p$. Consequently, using distributivity, we would have $\left(M_{i-}\right)^{\perp}=\bigvee_{j \neq i}\left(\left(M_{i-}\right)^{\perp} \cap\left(M_{j-}\right)^{\perp}\right)$ and, since $\left(M_{i-}\right)^{\perp}$ is joinirreducible, this would lead to $\left(M_{i-}\right)^{\perp} \subseteq\left(M_{j_{-}}\right)^{\perp}$, for some $j \neq i$. Then $M_{j-} \subseteq$ $M_{i-}$. But $M_{j} \nsubseteq M_{i}$ so $M_{i} \subseteq M_{j-}$. Hence we would have $M_{i} \subseteq M_{i-}$, contradicting $M_{i} \cap M_{i-}=(0)$. This completes the proof.

Remark. The preceding proposition shows that applying the operation $\left((\cdot)_{-}\right)^{\perp}$ interchanges the set of atoms and the set of join-irreducibles representing the underlying space irredundantly. Because of reflexivity it should not be surprising to observe then that ${ }^{\perp}\left(\left(\left(L_{-}\right)^{\perp}\right)_{-}\right)=L$ (where the second '-' on the left hand side is computed in $\left.\mathfrak{L}^{\perp}\right)$ holds if $L=M_{i}$ or $K_{j}$. In fact it holds for every $L \in \mathcal{Z}$. For, as observed earlier, $\left(\left(L_{-}\right)^{\perp}\right)_{-}=\left(\left(L_{-}\right)_{+}\right)^{\perp}$ so ${ }^{\perp}\left(\left(\left(L_{-}\right)^{\perp}\right)_{-}\right)=\left(L_{-}\right)_{+}$. Since $\left(L_{-}\right)_{+}=\bigcap\left\{M \mid M \in \mathfrak{L}\right.$ and $\left.M \nsubseteq L_{-}\right\}$it is clear that $L \subseteq\left(L_{-}\right)_{+}$. On the other hand, since $L \in \mathcal{Z}, L \nsubseteq L_{-}$so $\left(L_{-}\right)_{+} \subseteq L$. Hence $\left(L_{-}\right)_{+}=L$.

Theorem 3. Let $\mathfrak{L}$ be a finite distributive subspace lattice on a reflexive Banach space $X$. Let $\left\{M_{i} \mid 1 \leq i \leq p\right\}$ be the set of atoms of $\mathfrak{L}$ and let $X=\bigvee_{j=1}^{q} K_{j}$ be the unique irredundant representation of $X$ as a join of non-zero join-irreducible elements $\left\{K_{j} \mid 1 \leq j \leq q\right\}$. Let $m$ be the largest positive integer for which there exist subsets $I \subseteq\{1,2, \ldots, p\}$ and $J \subseteq\{1,2, \ldots, q\}$, each of cardinality $m$, such

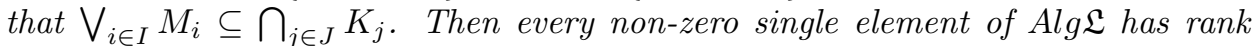
less than or equal to $m$. Also, for every $1 \leq n \leq m$, Alg $\mathfrak{L}$ contains a single element of rank $n$.

Proof. Let $S$ be a non-zero single element of Alg $\mathfrak{L}$. Since $X=\bigvee_{j=1}^{q} K_{j}, \overline{\mathcal{R}(S)}=$ $\bigvee_{j=1}^{q} S\left(K_{j}\right)$ where, by [4, Lemma 3.2], each $S\left(K_{j}\right)$ is at most one-dimensional. Thus $S$ has finite rank, $r$ say, and $\mathcal{R}(S)=\bigvee_{j=1}^{r} S\left(K_{j}\right)$, relabelling the $K_{j}$ if necessary. Similarly, using Proposition 2 and [4, Lemma 3.3], we obtain $\mathcal{R}\left(S^{*}\right)=$ $\bigvee_{i=1}^{r} S^{*}\left(M_{i-}\right)^{\perp}$, relabelling the $M_{i}$ if necessary, with $S^{*}\left(M_{i-}\right)^{\perp}$ one-dimensional, for every $1 \leq i \leq r$. That $\bigvee_{i=1}^{r} M_{i} \subseteq \bigcap_{j=1}^{r} K_{j}$ is proved exactly as in the final paragraph of the proof of Theorem 1 . Hence $r \leq m$.

Finally, let $1 \leq n \leq m$. Then, relabelling if necessary, we can suppose that $\bigvee_{i=1}^{n} M_{i} \subseteq \bigcap_{j=1}^{n} K_{j}$. Then conditions (1) and (2) as in the statement of Theorem 1 are satisfied since each $M_{i}$ is an atom of $\mathfrak{L}$ and, by Proposition 2 , each $\left(K_{j-}\right)^{\perp}$ is an atom of $\mathfrak{L}^{\perp}$. Thus $A l g \mathfrak{L}$ contains a single element of rank $n$ by Theorem 1 . This completes the proof.

Remark. With $\mathfrak{L}, p$ and $q$ as in the preceding theorem, the latter shows that every non-zero single element of $A l g \mathfrak{L}$ has rank less than or equal to $\min (p, q)$. Equality need not occur. For example, if $\mathfrak{L}$ is a complete atomic Boolean algebra with $p \geq 2$ atoms, then $q=p$, yet every non-zero single element has rank one. (However, if $\mathfrak{L}$ is the ordinal sum of two finite atomic Boolean algebras there does exist a single element of $\operatorname{Alg} \mathfrak{L}$ with rank equal to $\min (p, q)$ since in this special case $\bigvee_{i=1}^{p} M_{i}=$ $\bigcap_{j=1}^{q} K_{j}$. This fact was first observed by Lambrou [4, Theorem 5.3].) 


\section{REFERENCES}

1. T. Donnellan, Lattice theory, Pergamon Press, Oxford and New York, (1968). MR 38:2059

2. M. S. Lambrou, Approximants, commutants and double commutants in normed algebras, J. London Math. Soc. (2) 25 (1982), 499-512. MR 84f:47053

3. M. S. Lambrou, Automatic continuity and implementation of homomorphisms, (manuscript).

4. M. S. Lambrou, On the rank of operators in reflexive algebras, Linear Alg. \& Applic. 142 (1990), 211-235. MR 91k:47104

5. M. S. Lambrou and W. E. Longstaff, Spatiality of isomorphisms between certain reflexive algebras, Proc. Amer. Math. Soc. (4) 122 (1994), 1065-1073. MR 95b:47053

6. W. E. Longstaff, Strongly reflexive lattices, J. London Math. Soc. (11) 2 (1975), 491-498. MR 52: 15036

7. G. N. Raney, Tight Galois connections and complete distributivity, Trans. Amer. Math. Soc. 97 (1960), 418-426. MR 22:10928

8. J. R. Ringrose, On some algebras of operators II, Proc. London Math. Soc. (3) 16 (1966), 385-402. MR 33:4703

Department of Mathematics, The University of Western Australia, Nedlands, Western Australia 6907, Australia

E-mail address: longstaf@maths.uwa.edu .au

E-mail address: oreste@maths.uwa.edu.au 